\title{
Simulator for the Receiver-end of an Optical Communication System
}

\author{
Marco Costa Pereira ${ }^{1}$, Maria João Martins ${ }^{2}$, Helena Rocha ${ }^{2}$, Renata Viegas ${ }^{2}$ \\ ${ }^{1}$ Esc. Sec. D. Luisa de Gusmão, Rua da Penha de França, mcpereira_80@yahoo.com \\ ${ }^{2}$ Instituto Superior Técnico, Av. Rovisco Pais, pcjoaom@mail.ist.utl.pt
}

\begin{abstract}
The merge of the computer and telephone networks that marked the end of the $\mathrm{XX}^{\text {th }}$ century, will be followed by the triple integration of the computer, telephone and digital interactive television networks. The need for higher bandwidth in a global scale for the transmission of real-time video, will change the main role of optical communication systems from the high bit-rate international and intercontinental digital networks to local area networks, that can also benefict from the immunity of such systems to electromagnetic interference. Therefore it is necessary to increase the proficiency and broaden the experience of students of electrical engineering in optical systems, not just by higher workload on the cost and maintanence demanding equipments of university and high school optical labs. The computer simulator can constitute an answer for a portable, safe and low cost optical lab and it that can prepare students to the experimental procedures in an optical lab. This paper presents the framework of a functional simulator for the receiver of an optical communication system. The blocks of such systems are represented by behavioural multilevel models with input, output and internal parameters.
\end{abstract}

Keywords: simulation/experiments, functional simulator, behavioural multilevel models

\section{INTRODUCTION}

The optical communication system can be divided into three main function blocks: the transmitter, the optical fibre/cable and the receiver. As presented in figure 1, both the transmitter and the receiver can have several sub-blocks.

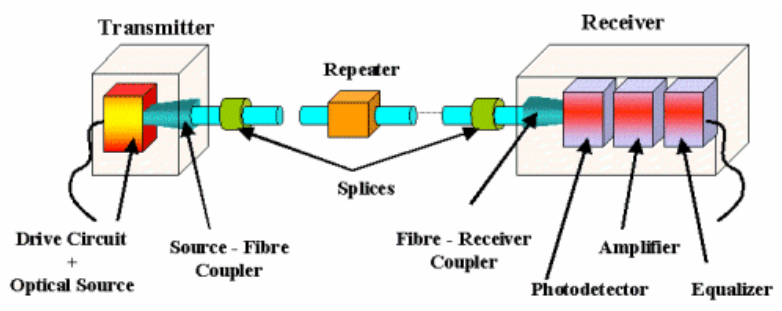

Figure 1-Analogue optical communication system.

The transmitter transforms the electrical signal into an optical signal to be sent into the optical fibre by a coupler. The optical source (usually a LED or a laser) has to assure that the maximum optical power is transmitted into the fibre with very small losses.
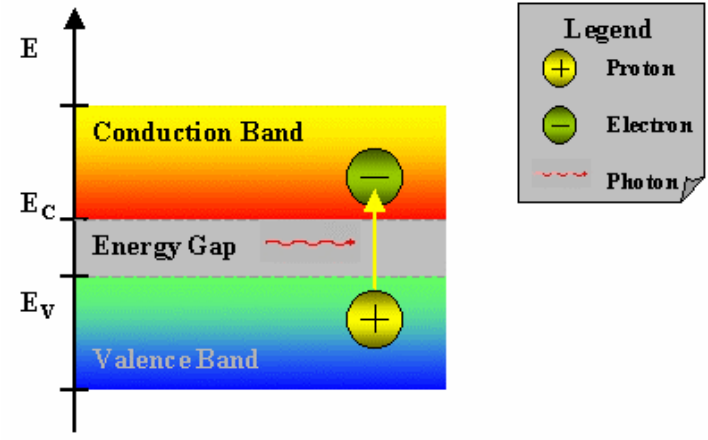

Figure 2-Photodetector working principle.
For analogue optical systems the linearity is also an important parameter. In the receiver, the noise enbedded optical signal is translated into an electrical signal by the photodetector (figure 2). The limitations of the photodetector can be overcomed by the use of an amplifier, filter and equalizer to obtain an analogue signal that matches as much as possible the transmitted input signal.

Most of the optical systems designed and installed in the last century are digital, used mainly in high bit-rate international networks. Nowadays due to the integration of all communication networks: computer global network - the internet, mobile and fixed phone network and television networks, the need for bandwidth in local areas will increase to higher values thus leading to the design of local digital optical networks in parallel with non guided communication systems already in operation. So, to the blocks illustrated in figure 1, it should be added the analoguedigital (A/D) converter before the transmitter and the digital-analogue $(\mathrm{D} / \mathrm{A})$ converter after the elimination of noise by the filter. Other blocks such as the modulator and demodulator, the multiplexer and the demultiplexer can also be added for high bit-rate networks.

The simulator design principles and structure for the receiver of an optical communication system are presented in section two. The modelling procedures and simplified examples for the PIN photodetector and the $\mathrm{D} / \mathrm{A}$ signal converter are presented in section three. Two example of the use of the simulator are presented in section four. Conclusions are presented at section five. 


\section{THE SIMULATOR}

\subsection{Principles for the design}

Nowadays, the tools available to a teacher were extended from the written/spoken word, to video and sound. The computer allowed the integration of these tools into a complete learning environment [1, 2]. Sound and video animations are particularly useful if properly used, to give further incite on the explanation of a concept. However, a simulator can provide a low cost and safe "laboratory environment" for several experiments, giving the student a full grasp of the studied subject. In a computer simulator, the student can test several situations, using time analysis and parameter variation, in a user-friendly environment. There are five major concerns in the development of a simulator: the accuracy of the models and the simulation time to achieve accurate result values, the number and variety of models for several available components, the existance of a tutorial guide for the simulation of the block or system of blocks and an intuitive easy-to-use user-friendly software environment. The accuracy of the models of the blocks that constitute the receiver-end of an optical communication system is the most important characteristic in a simulator. The results must be as accurate and precisely as possible, considering the pedagogic or even scientific objectives of the simulator. One of the major drawbacks of simulators is the high simulation time, especially for analogue complex circuits. The early SPICE simulators [3-7] are inadequate for the complexity of analogue microsystems, and can not simulate p.e. the phaselocked loop circuit because the voltage-controlled oscillator (VCO) frequency is much higher than the loop frequency [7]. Some techniques were developed to reduce simulation time, like iterative relaxation methods, that can be generalized into waveform relaxation methods that speed up timing simulation for discrete signal circuits [7]. Due to its pedagogic purpose this simulator is based on functional multilevel models [8]. The number and type of models in the library should assure a significant number of possible system simulations with several variants. A guide to the most important features in a optical communication system such as noise at the input caused by the attenuation and dispersion of the signal in the optical fibre, the coupling fibre-photodetector, the response time of the blocks $[9,10]$, the noise introduced by the several blocks, the influence of the input dynamic range, the change in parameters of the recuperation filter and/or the equalizer and the clock jitter, will be part of the simulator. The student should be guided in experimenting with the simulator by changing only a few key parameters to better understand the influence of those parameters on the system performance. This is also the main reason for the use of a simulator as a learning tool - to better understand the influence of a design parameter in the entire system. Finally, the simulator should present the results, in a graphical interface providing the best visualization of the signals at the input of every block and at the output of the system. This user-friendly environment should be WYSIWYG.

\subsection{Structure of the Simulator}

The simulator can be divided into three main software tools:

1. The model libraries.

2. The simulator.

3. The tutorial tool.

The macromodels package consists of several models of the blocks that can define the receiver-end of a digital optical communication system. The analogue system although optional, it has pedagogic advantages in the study of the influence of signal distortion in phase, amplitude and frequency from the photodetector through each of the following blocks. Each model has a set of input signals and a set of output signals and internal parameters. The input signals can be defined by the user, or restricted by the previous block. The simulator defines the input vectors based on the input signals restrictions in range and simulation sampling time. The input vectors are propagated through the block or set of blocks, by the use of mathematical equations and an output vector for each block is obtained. After the block selection, the tutorial tool gives a set of blocks that are compatible with the input, and a set of blocks that are compatible with the output (figure 3). The tutorial tool also guides the user throughout the definition of input and block parameter restrictions to assure the viability of the simulation. It has also predefined examples that can be used in a guided computer-aided-learning environment.

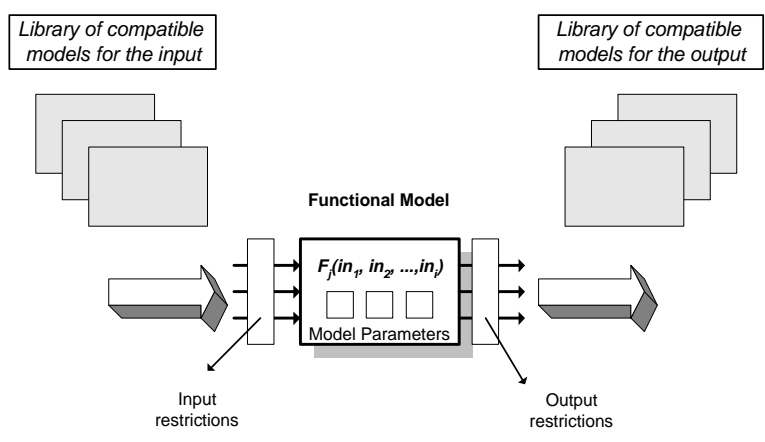

Figure 3-Framework of the simulator.

The modular structure of the blocks allows that each block be analysed individually or as a part of the receiver subsystem. As mention before, the influence of the parameters in a block can thus be evaluated 
through the entire subsystem. A different approach was followed by [11], where students developed their own laser model and study the optical signal through the optical communication system.

\section{THE BEHAVIOURAL MODELS}

\subsection{Models description}

Each model has three levels that express the outputs as functions of the several inputs, with higher degrees of complexity. The second and third levels of representation consider second-order effects and noise influences. The major drawbacks of behavioural models listed in [6], namely in the simulation of frequency domain performance and noise performance [8], will be minimized by using the same mathematical approach. The multilevel behavioural models are most suitable for time-domain and parameter sweep analysis.

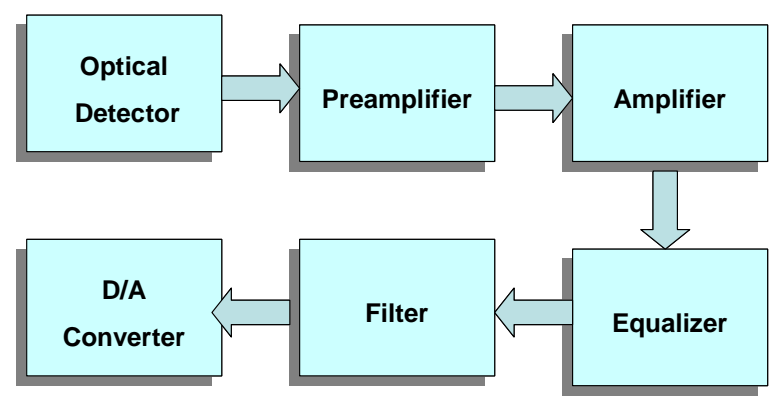

Figure 4-An simplified optical communication system.

A typical optical system is presented in figure 4 . According to the design principles of the simulator presented earlier, the signal propagates from the input vectors of the first block through the entire system, generating timed output vectors.

\subsection{The simplified PIN photodiode model}

The PIN photodiode is a device that uses incident photons to generate a current of electrons and holes. The output primary current of the photodiode is given by [9]:

$I_{p}=\frac{q}{h f} P_{0}\left(1-e_{s}^{-\alpha(\lambda) * w}\right)\left(1-R_{f}\right)$

The symbol $\mathrm{P}_{0}$ stands for the incident power and:

$P(w)=P_{0}\left(1-e_{s}^{-\alpha(\lambda) * w}\right)$

Represents the absorved power without considering reflected rays, in the direction $\mathrm{w}$. The term $\left(1-\mathrm{R}_{\mathrm{f}}\right)$ represents the power loss due to reflection, thus $R_{f}$ is a device reflection coefficient. The two constants in (3.2.1) are the electron charge $(q)$ and the Plank's constant $(h)$. On a simplistic view, the formula (3.2.1) can be seen as a relation between the generated electron charge by time unit, corresponding to the IS definition of electrical current intensity.

$I_{p}=q P(w) / E(f)=q / \Delta t$

The relation between the absorved power and the energy of a photon, $E(f)=h f$, defines the output primary current of the photodiode. The absorved power depends on the $\alpha_{s}(\lambda)$ absortion coefficient of the juntion(s) material(s). The primary responsivity of the device is given by an output/input relation:

$R_{0}=\frac{I_{p}}{P_{0}}=\frac{\eta q}{h f}$

Where $\eta$ is given by:

$\eta=\frac{I_{p} / q}{P_{0} / h f}$

The total output current is given by:

$I_{M}=M \cdot I_{p}$

The factor $M$ is a statistical average multiplication coefficient of the photodiode. For the PIN photodiode is equal to one. This set of equations can represent the first level model of the photodiode, with the input parameters of incident direction $(\mathrm{w})$, incident power $\left(\mathrm{P}_{0}\right)$ and incident radiation frequency $(\mathrm{f})$. Other input parameters will be considered for the second and third order models: noise power density or signal to noise ratio of the input signal, coupling power loss or coupling power efficiency and noise generated in the photodetector. Considering that the noise sources are uncorreled the total noise current density is given by [9]:

$<\mathrm{I}_{\mathrm{N}}^{2}>=<\mathrm{I}_{\mathrm{Q}}^{2}>+<\mathrm{I}_{\mathrm{DB}}^{2}>+<\mathrm{I}_{\mathrm{DS}}^{2}>+<\mathrm{I}_{\mathrm{T}}^{2}>$

The expression (3.2.7) can be calculated by:

$<\mathrm{I}_{\mathrm{N}}^{2}>=2 \mathrm{q}\left(\mathrm{I}_{\mathrm{P}}+\mathrm{I}_{\mathrm{D}}\right) \mathrm{BM}^{2} \mathrm{~F}(\mathrm{M})+2 \mathrm{qI}_{\mathrm{L}} \mathrm{B}+\frac{4 k_{B} T}{R_{L}} \mathrm{~B}$

The last term of the expression (3.2.8) refers to the termal noise generated by the output resistance of the photodetector. The term: 
$<\mathrm{I}_{\mathrm{DS}}^{2}>=2 \mathrm{qI}_{\mathrm{L}} \mathrm{B}$

Represents the influence of the superficial leakage dark current of the photodiode. The term:

$<\mathrm{I}_{\mathrm{Q}}^{2}>=2 \mathrm{qI}_{\mathrm{P}} \mathrm{BM}^{2} \mathrm{~F}(\mathrm{M})$

Represents the noise associated to the random movement of carriers in the semiconductor material, where $\mathrm{B}$ is the frequency bandwidth and $\mathrm{F}(\mathrm{M})$ is a function the multiplication factor, dependent of the junction(s) material(s) and dimensions. Finally the term:

$<\mathrm{I}_{\mathrm{DB}}^{2}>=2 \mathrm{qI}_{\mathrm{D}} \mathrm{BM}^{2} \mathrm{~F}(\mathrm{M})$

Represents the influence of the primary dark current of the photodetector given by $I_{D}$.

\subsection{The simplified D/A converter model}

The digital-analogue (D/A) converter is a block of an optical digital communication system. To define a functional model for the D/A converter $[12,13]$, the authors considered:

- $\quad$ Input parameters: resolution $(\mathrm{N})$, voltage levels for the $n$ digital inputs and sampling frequency.

- $\quad$ Output parameter: output voltage.

As input restrictions the authors considered the voltage threshold or thresholds for the digital inputs, that define the " 1 " and " 0 " levels, and that the number of inputs must be equal or smaller than the value of the resolution $(\mathrm{N})$. The output restrictions are defined as the output dynamic range and the output maximum frequency.

The input restrictions are used to define the sequence of the input binary digits: $b_{1}, b_{2}, \ldots, b_{N}$. The input of the $\mathrm{D} / \mathrm{A}$ converter can be defined as:

$B_{I N}=\sum_{k=1}^{N} 2^{k-1} b_{k}=2^{0} b_{1}+2^{1} b_{2}+2^{2} b_{3}+\ldots+2^{N-1} b_{N}$

In (3.3.1), $b_{1}$ defines the least significant bit (LSB) and $\mathrm{b}_{\mathrm{N}}$ defines the most significant bit (MSB). The output voltage of an unipolar D/A converter, for the model in the first level can be defined as:

$v_{\text {OUT }}=k_{V} \frac{V_{R E F}}{2^{N}} B_{I N}=k_{V} \frac{V_{R E F}}{2^{N}} \sum_{k=1}^{N} 2^{k-1} b_{k}$
In the expression (3.3.2), $V_{R E F}$ is the reference voltage that defines the output dynamic range and $k_{V}$ is an adaptation factor, near 1 . Thus the voltage that corresponds to a LSB is:

$v_{L S B}=\frac{V_{R E F}}{2^{N}}$

So the expression $B_{I N}$ represents the number of LSBs of the input code. The output is obtained by a simple product of that number by the voltage of a LSB. Both $V_{R E F}$ and $k_{V}$, are internal model parameters. The simplicity of the first level will allow the explanation of the influence of the resolution and reference voltage on the output.

For the second and third levels of the functional model, among other parameters, the authors considered four static performance limitations and one dynamic limitation.

The four static performance limitations are:

1. The offset $\left(v_{O S}\right)$ and gain $\left(f_{g}\right)$ errors:

$v_{O U T}=f_{g} k_{V} \frac{V_{R E F}}{2^{N}} \sum_{k=1}^{N} 2^{k-1} b_{k}+v_{O S}$

2. The integral and differential nonlinearity errors:

$v_{\text {OUT }}=f_{g} k_{V} \frac{V_{R E F}}{2^{N}} \sum_{k=1}^{N}\left(2^{k-1} b_{k} \pm \Delta_{k}\right)+v_{O S}$

The equations presented so far don't have the time as one of its variables. For simplicity, the equations are considered only valid at every $\mathrm{kT}_{\mathrm{s}}$ seconds, where $\mathrm{T}_{\mathrm{s}}$ is the sampling period. The $\mathrm{D} / \mathrm{A}$ settling time defined as the time the output takes to settle within a percentage of variation $(\varepsilon)$ of the final output value $\left(v_{\text {OUTF }}\right)$ is given by [12]:

$\Delta t_{s}=-\tau \ln \left(v_{\text {OUTF }} \frac{\varepsilon}{100}\right)$

In (3.3.6), $\tau$ is the time constant dependent on the output resistance and capacitance. The settling time is measured at the sampling instant $\mathrm{kT}_{\mathrm{s}}$.

4 AN EXAMPLE FOR THE PHOTODETECTOR The simulator window for the photodetector is presented in figure 5. The user can choose the type of photodetector, mainly its semiconductor junction material(s) and the level of the model used, on top of the window. At the center of the window the student can define the internal parameters of the studied 
element. Input vectors are given in the left part of the window and output vectors are showed, during simulation, in the right part of the window. A similar structure is presented for the simulation of the D/A converter in figure 6 .

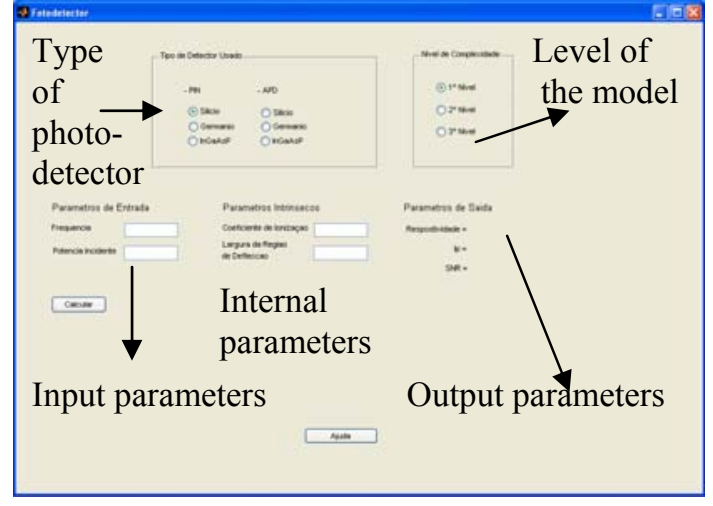

Figure 5 - Window of the simulator for the photodetector.

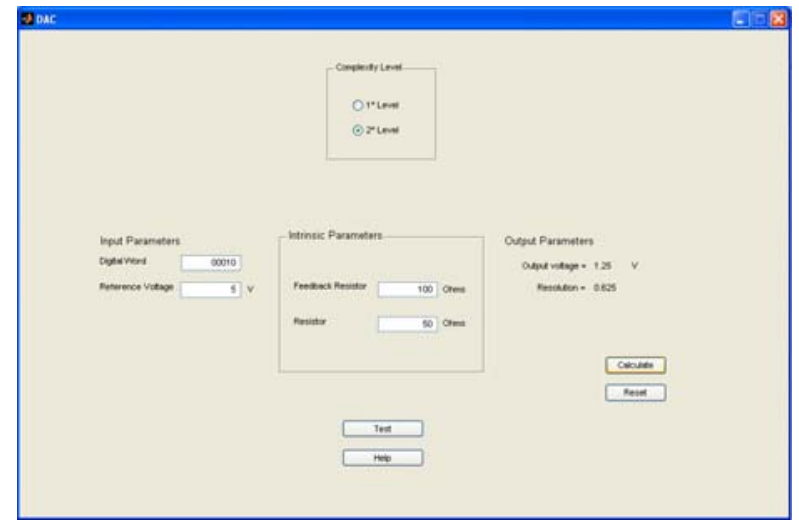

Figure 6-Window of the simulator for the D/A converter.

\section{CONCLUSIONS}

The definition of the most important design factors led to the development of a simulator for the receiver-end of a optical communication system framework. The main pedagogic function of this simulator beneficts from the functional multilevel models for the major blocks that constitute the receiver. Each model has three levels of accuracy. The second and third levels allow the simulation of second-order and noise effects. Simplified examples of multilevel models were presented for the PIN photodetector and the D/A converter. The simulator windows for the photodetector and the D/A converter were decribed.

\section{Bibliography}

1.Maria J. Martins, Marco Pereira, "Integrated Approach to Science Teaching by using ICT and CBL", in Proceedings of the $15^{\text {th }}$ International
EAEEIE Conference, Sofia, Bulgaria, $27^{\text {th }}-29^{\text {th }}$ of May of 2004.

2. M. J. Martins, M. C. Pereira, S. Kocjancic, "Sensorcomputer interface", web-based course developed under the EU project ComLab-Sci.

3.L. Nagel, "SPICE2: A computer Program to Simulate Semiconductor Circuits", $\mathrm{PhD}$ thesis, University of California, Berkeley, May 1975.

4.T.L. Quarles, "Analysis of Performance and Convergence Issues for Circuits Simulation", $\mathrm{PhD}$ thesis, University of California, Berkeley, 1989.

5. B. Johnson, T. Quarles, A.R. Newton, D.O. Pederson and A. Sangiovanni-Vincentelli, "SPICE3 Version 3e User's Manual", University of California, Berkeley, April 1991.

6.H. Chang, E. Charbon, U. Choudhury, A. Demir, E. Felt, E. Malavasi, A. Sangiovanni-Vincentelli, I. Vassiliou, "A top-down constraint-driven methodology for analog integrated circuits", Kluwer Academic Publishers, 1997.

7.R. Saleh, S-J Jou and A.R. Newton, "Mixed-Mode Simulation and Analog Multilevel Simulation", Kluwer Academic Publishers, Boston, MA, 1994.

8.E. Liu, "Analog Behavioral Simulation and Modelling", $\mathrm{PhD}$ thesis, University of California, Berkeley, 1993.

9. M. J. Martins and M. C. Pereira, "Optoelectronics", web-based course developed under the EU project ComLab-Sci.

10. Marco Pereira, Maria J. Martins, "Experiments in an Optoelectronics Web-based Course", in Proceedings of the $15^{\text {th }}$ International EAEEIE Conference, Sofia, Bulgaria, $27^{\text {th }}-29^{\text {th }}$ of May of 2004.

11. Diaa Khalil and Smail Tedjini, "Teaching Laser Dynamics and Optical Communication Systems Using a Standard System Simulator”, IEEE, 2000.

12. Marco Pereira, Bernardo Gorjão Henriques "Memorandum: ALDAC - Automatic Module and Layout Generation of High-Speed CMOS D/A Converters ", for the project ESPRIT 8030 - ALCD (Analogue Libraries for CMOS Digital Technologies), June 1995.

13. David A. Johns and Ken Martin "Analog Integrated Circuit Design”, John Wiley \&Sons Inc., 1997. 